\title{
Improving a Terahertz Time-Domain Spectroscopy Apparatus Using Neodymium Magnets
}

\author{
Kaori Yano ${ }^{1}$ and Toshiaki Hattori ${ }^{1, a^{*}}$ \\ ${ }^{1}$ Graduate School of Pure and Applied Sciences, University of Tsukuba, Tsukuba, Ibaraki 305-8573, \\ Japan \\ ahattori@bk.tsukuba.ac.jp
}

Keywords: terahertz time-domain spectroscopy, InAs, neodymium magnet

\begin{abstract}
The signal-to-noise ratio obtained from terahertz time-domain spectroscopy is significantly affected by the low available power of terahertz waves. We constructed a terahertz-wave source with emission power enhanced by a magnetic field. The emitter is composed of an InAs wafer and two neodymium magnets. The emitter was irradiated by femtosecond laser pulses. The data quality of terahertz spectroscopic measurements was evaluated, and reduction of error in the data obtained due to the terahertz power enhancement was observed.
\end{abstract}

\section{Introduction}

Terahertz time-domain spectroscopy (THz-TDS) is a very powerful and widely applicable measurement method which can evaluate both the absorptive and dispersive properties of target materials. Detection of terahertz waves, which are radiation with frequencies between $0.03 \mathrm{to} 12 \mathrm{THz}$, is generally difficult, and this difficulty prohibits wider application of this region of electromagnetic radiation. Terahertz-wave emission from a surface of InAs crystal under irradiation of ultrashort light pulses provides us with a simple emitter that can be widely used although the obtained power of the terahertz waves is rather weak. It has been reported that the emitted power can be enhanced by application of magnetic field [1]. By using neodymium magnets, which are compact and powerful magnets, becoming available recently, we can apply sub-tesla magnetic field easily to the emitter crystal without significant modification of existing setup.

In this study, we have incorporated neodymium magnets into an existing THz-TDS apparatus, and achieved enhancement of the emitted terahertz field. We evaluated the performance of the spectroscopic measurements, and observed improvement of the signal-to-noise $(\mathrm{S} / \mathrm{N})$ ratio due to the terahertz power enhancement.

\section{Experimental}

In this study, terahertz waves were generated by irradiating a (111) $p$-type InAs wafer (carrier density: $2-4 \times 10^{17} \mathrm{~cm}^{-3}$ ) by $800 \mathrm{~nm}$ mode-locked Ti:sapphire laser pulses [2, 3]. The incident angle of the laser light was $45^{\circ}$. It has been reported that the dominant mechanism of the terahertz-wave emission from the surface of InAs is photo-Dember effect [2]. As shown in Fig. 1, terahertz waves are emitted by the dipole moments created by photo-induced transient electric current. Without magnetic field, the direction of the dipole moment is normal to the emitter surface, and major part of the emitted power is reflected into the crystal at the surface. Assuming the refractive index of InAs at terahertz frequency region as 3.84 [4], the transmittance of the terahertz waves through the surface from InAs to the air is calculated as a function of incident angle as shown in Fig. 2. By application of magnetic field, the direction of the dipole moment can be tilted, which increases the portion of terahertz radiation with smaller incident angle to the surface. When the laser pulses are incident to the semiconductor with a large angle, and terahertz radiation emitted around the mirror reflection direction is collected. Thus the magnetic field applied in the direction shown in Fig. 1 is known to enhance the terahertz emission power [5]. 


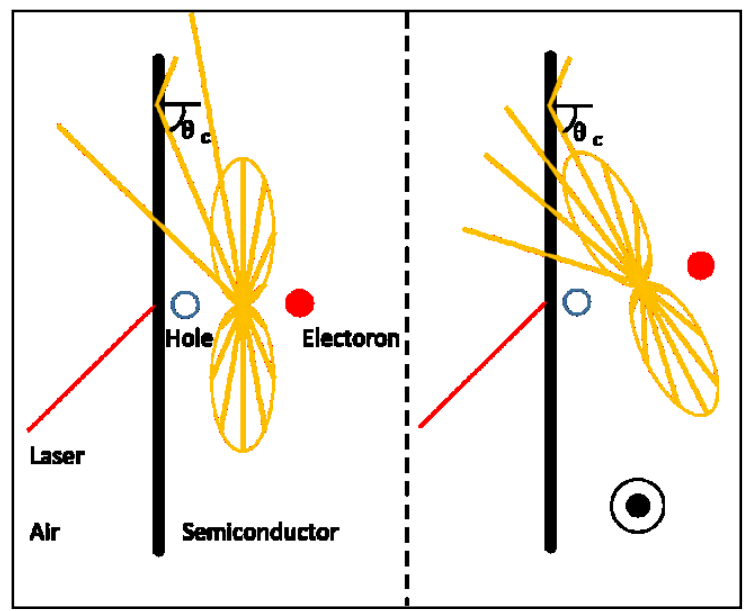

Figure 1. Radiation patters of terahertz waves from an irradiated semiconductor surface without magnetic field (left), and with magnetic field (right). The applied magnetic field is assumed in the direction from back to front of the paper.

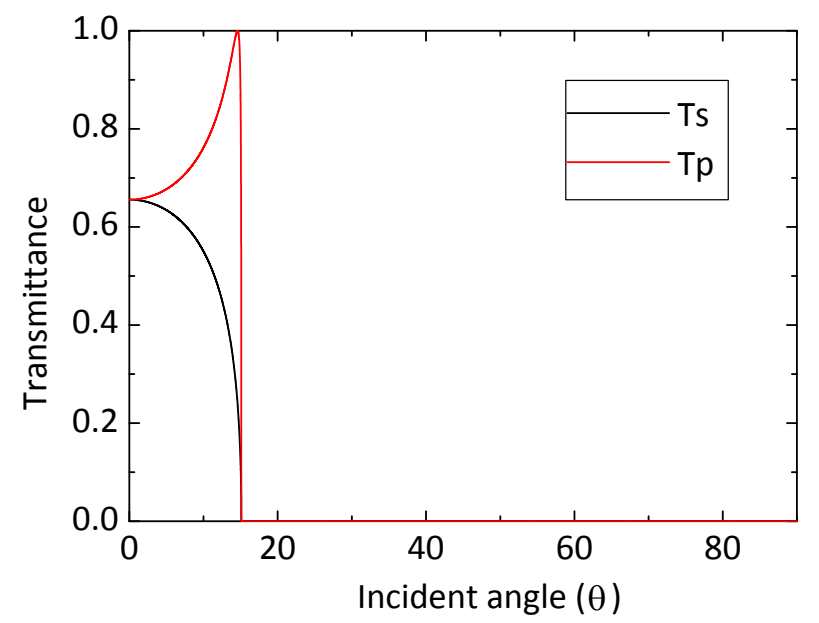

Figure 2. Transmittance of terahertz waves from within InAs to the air as a function of incident angle, calculated assuming a refractive index of 3.84. Black curve is for $s$-polarization and red for p-polarization.

We used a pair of neodymium magnets of a cylinder shape with a diameter of $9 \mathrm{~mm}$ and a length of $20 \mathrm{~mm}$. An iron yoke was used to hold them, as shown in Fig. 3. The spacing between the magnets was $6 \mathrm{~mm}$. The magnetic field at the center of the gap was $560 \mathrm{mT}$, which was measured by a gaussmeter. An aluminum magnet holder, as shown in Fig.3, was fabricated with a portion that can hold the InAs crystal in the gap of the magnets.

Here, we consider the cyclotron motion of the electrons under the magnetic field applied. When a magnetic field $B$ is applied to an electron having a charge $q$ and mass $m^{*}$ and moving at a velocity of $v$, the electron undergoes cyclotron motion with a radius of $r$ under the equation of motion: $m^{*} v^{2} / r=q v B$. The period of the cyclotron motion $T$ is expressed as $T=2 \pi m^{*} / q v$. On the other hand, the scattering time of electron $\tau$ is related to the mobility $\mu$ as $\tau=\mu \mathrm{m}^{*} / q$. Using the values of effective mass of 0.023 , mobility of 20,000 to $33,000 \mathrm{~cm}^{2} \mathrm{~V}^{-1} \mathrm{~s}^{-1}$ (for carrier density of $10^{15}-10^{16}$ $\mathrm{cm}^{-3}$ ) [6], and the magnetic field of $560 \mathrm{mT}$, we obtain $T=1.5 \mathrm{ps}$ and $\tau=0.26$ to $0.43 \mathrm{ps}$. The actual value of the scattering time should be shorter than this since the present carrier density is much larger. From this discussion, we can conclude that the electron scattering time is much shorter than the 
cyclotron motion period, and therefore, tilting of the dipole direction due to the magnetic field is not too much.
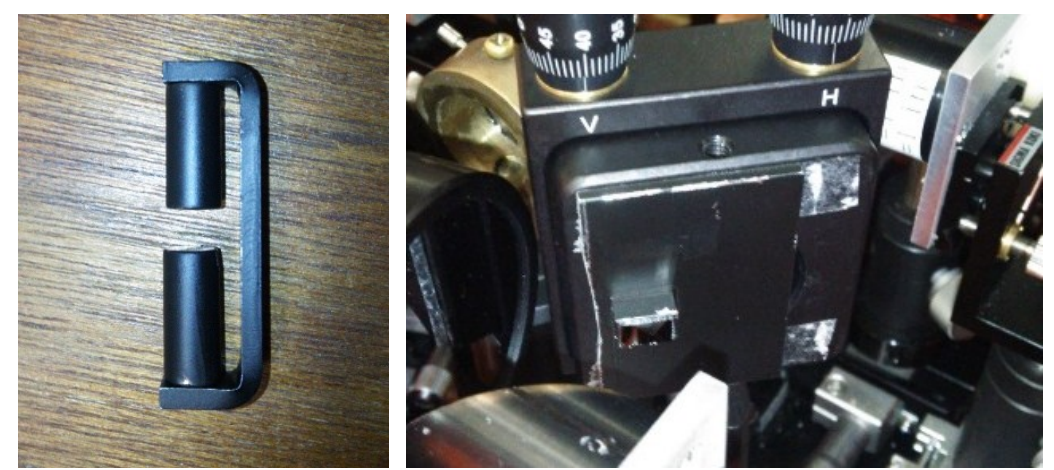

Figure 3. (left) Photo of the neodymium magnets attached to the yoke. (right) Photo of the InAs emitter attached to the magnet holder.

\section{Experimental}

The THz-TDS apparatus used in the present study is essentially the same as previously described [3] except for the magnetic field application. The terahertz field was detected by an electro-optic sampling method using a ZnTe crystal. We evaluated the data quality by measuring the spectra of the terahertz radiation and also the absorption and refractive index spectra of water. For each set of data, three configurations of the emitter were used for comparison, i.e., without a magnet field, a magnetic field applied in the top-to-bottom direction, and a magnetic field in the opposite direction. The whole setup was kept in dried nitrogen atmosphere, and the temperature was kept at $20-21^{\circ} \mathrm{C}$.

\section{Results and Discussion}

Figure 4 shows the obtained waveforms of terahertz waves. The terahertz field observed from the emitter with a magnetic field applied was about 1.5 times larger than that without a magnetic field, which corresponds to 2.25 times enhancement of terahertz power. We observed a delay of $0.4 \mathrm{ps}$ in the peak position when a magnetic field is applied, which is similar to the behavior observed previously [7]. When the magnetic field is inverted, the observed terahertz field was also inverted, which means that dipole moment is inverted by the applied magnetic field.

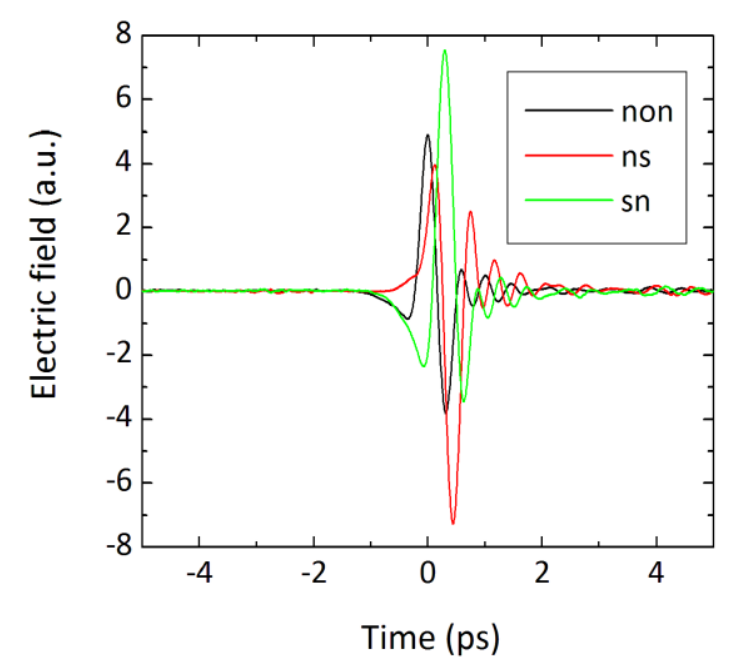

Figure 4. Waveforms of terahertz waves measured in three configurations of the emitter: waveform obtained without a magnetic field applied (non), with a magnetic field of $560 \mathrm{mT}$ applied in the top-to-bottom direction (ns), and with a magnetic field of $560 \mathrm{mT}$ applied in the bottom-to-top direction (sn). 


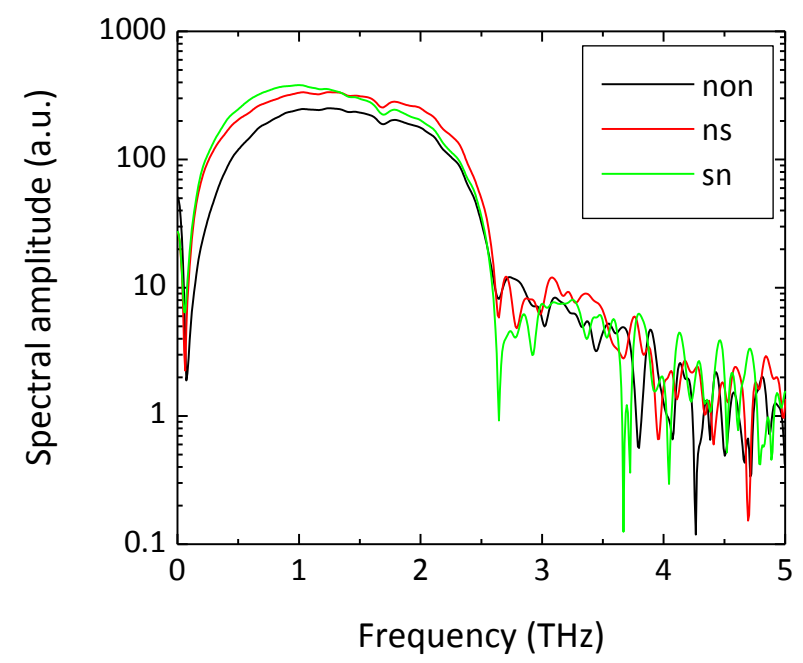

Figure 5. Amplitude spectra of the terahertz waveforms shown in Fig. 4.

The amplitude spectra of these waveforms are shown in Fig. 5. The spectra exhibit amplitude enhancement in the frequency range between 0.1 and $2.5 \mathrm{THz}$ by the application of the magnetic field to the emitter crystal, and the enhancement is more pronounced in the lower frequencies.

For the evaluation of $\mathrm{S} / \mathrm{N}$ ratio of spectroscopic data, transmission measurements of water were carried out using the three emitter configurations. The sample cell used in the measurement was the same as the previous our report [3]. The liquid sample with a thickness of $0.1 \mathrm{~mm}$ is inserted between two silica glass windows with a thickness of $1 \mathrm{~mm}$. For a reference, a pair of the same windows without water was used. Ten sets of data were collected alternatively from the sample and the reference, and the refractive index and the absorption coefficient of water was obtained. Standard deviations of the refractive index and the absorption coefficient were calculated for evaluation of the signal quality.

Figure 6 shows the spectra of refractive index and absorption coefficient of water obtained after averaging the results of the ten data sets. The spectra are very noisy in the frequency region below 0.5 $\mathrm{THz}$ and above $2.2 \mathrm{THz}$, which is due to the very low power of the terahertz waves in these frequency regions.

Figure 7 shows the standard deviation calculated from the ten data sets of the refractive index and absorption coefficient of water. Without a magnetic field, we achieved standard deviation of refractive index less than 0.01 in the frequency region of 0.5 to $2.2 \mathrm{THz}$. On the other hand, with the top-to-bottom magnetic field and bottom-to-top field, the region was extended to $0.3 \mathrm{THz}$ and 0.2 $\mathrm{THz}$, respectively. The result of absorption coefficient also shows extension of low-noise region to lower frequencies by the application of a magnetic field.
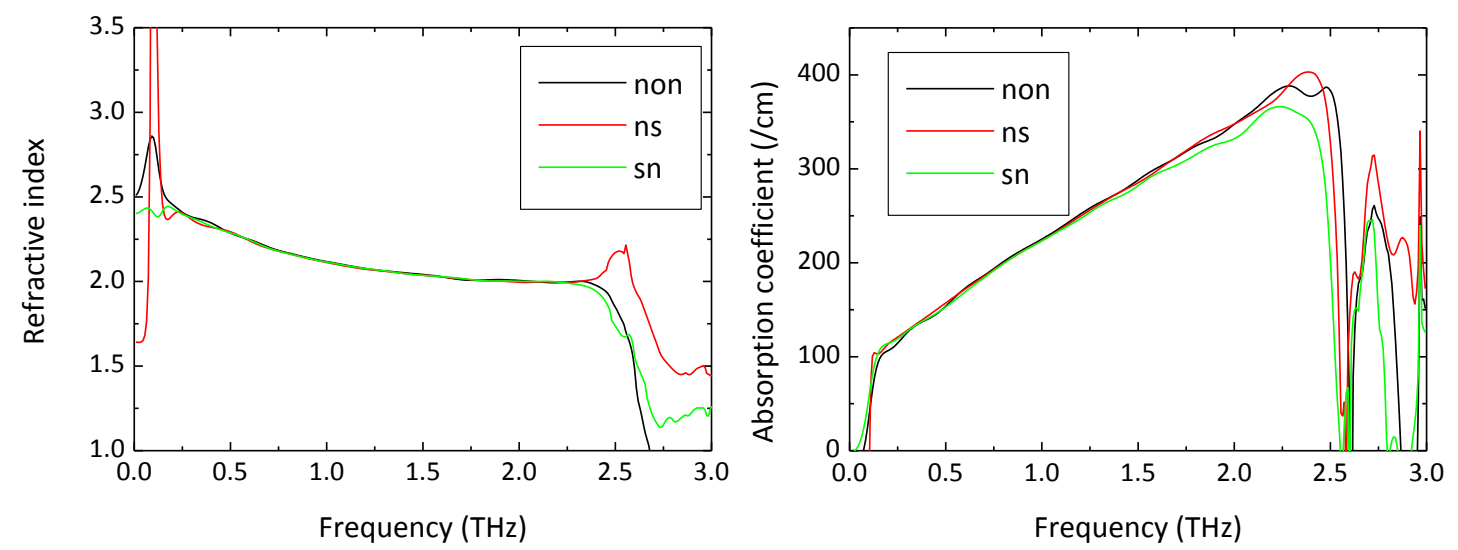

Figure 6. Averaged refractive index (left) and absorption coefficient (right) of water obtained using the three emitter configurations. 

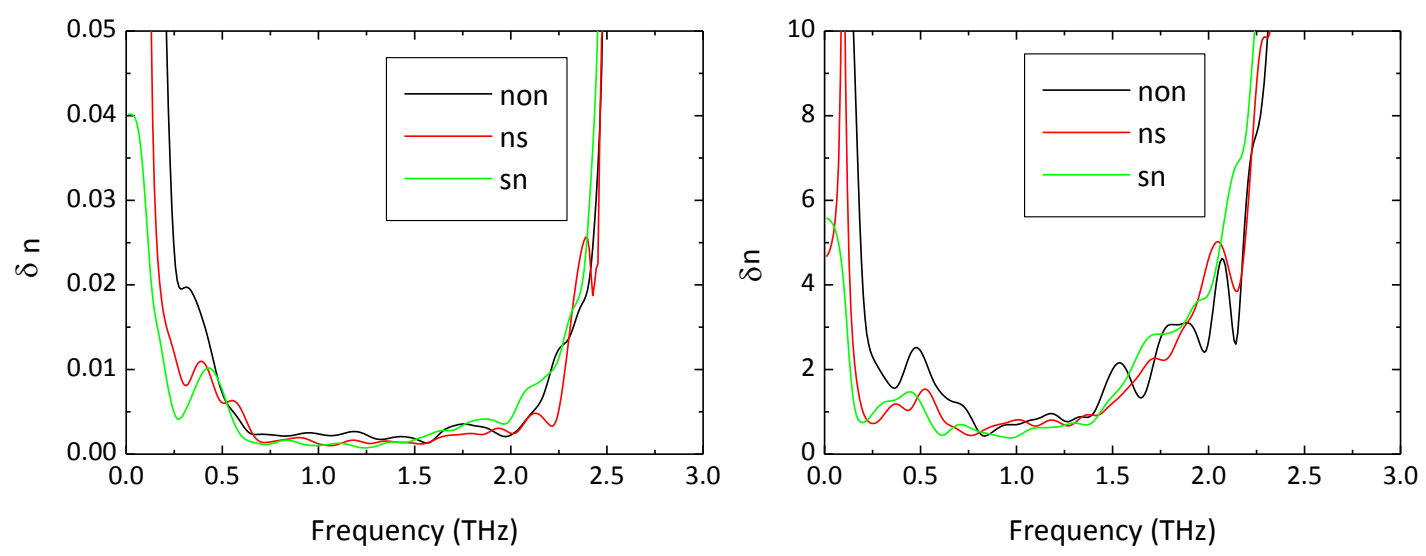

Figure 7. Standard deviation of the measured refractive index (left) and absorption coefficient (right).

As shown in Fig. 5, the terahertz amplitude enhancement due to magnetic field application is pronounced especially in the frequency region of 0.2 to $1.5 \mathrm{THz}$, which corresponds to the region where reduction of the error in the refractive index and absorption coefficient.

\section{Conclusions}

We have constructed a terahertz-wave emitter consisting of an InAs crystal with a pair of neodymium magnets, which can apply a magnetic field of $560 \mathrm{mT}$ to the InAs. This improvement of the emitter part of the terahertz time-domain spectroscopy apparatus was carried out without any modification of the other parts of the whole optical setup. We observed 2.25 times enhancement of the emitted terahertz power in the time domain waveforms by the magnetic field application. In the spectra, 0.2 to $1.5 \mathrm{THz}$ region showed pronounced amplitude enhancement. Transmission spectroscopic measurements of water were performed and the error in the obtained refractive index and absorption coefficient was evaluated, which shows improvement in the data quality in the corresponding frequency region.

\section{References}

[1] N. Sarukura, H. Ohtake, S. Izumida, and Z. Liu, J. Appl. Phys. 84 (1998) 654.

[2] R. Adomavičius, A. Urbanowicz, G. Molis, and A. Krotkus, Appl. Phys. Lett. 85 (2004) 2463.

[3] K. Aoki, K. Shiraki, and T. Hattori, Appl. Phys. Lett. 103 (2013) 173704.

[4] R. Mendis, M. L. Smith, L. J. Bignell, R. E. M. Vickers, and R. A. Lewis, J. Appl. Phys. 98 (2005) 126104.

[5] H. Ohtake, S. Ono, M. Sakai, Z. Liu, T. Tsukamoto, and N. Sarukura, Appl. Phys. Lett. 76 (2000) 1398.

[6] O. Madelung, "Semiconductors: Data Handbook” 3rd ed., Springer, Berlin, (2003).

[7] C. Weiss, R. Wallenstein, and R. Beigang, Appl. Phys. Lett. 77 (2000) 4160. 